\title{
Digital Image Processing ApProach to FINGERPRINT AUTHENTICATION
}

\author{
RAKUN, J.; BERK, P.; STAJNKO, D.; OCEPEK, M. \& LAKOTA, M.
}

Abstract: In this work, we present a digital image processing approach to fingerprint based authentication, which could be a part of an embedded robotic system. It uses digitalized fingerprint images that are made up by unique distribution of minutiae features such as ridge endings, ridge bifurcations, spurs, delta and cores. In order to minimize the computational requirements for an embedded system, the approach transforms each digitalized image to a distinct 12 dimensional 8-bit feature vector, which is accomplished with the help of gradients and wavelet transform. The feature vectors are then simply compared and used to compute a MSE that serves as a similarity measure. We have analysed 30 randomly selected examples and results show the approach is suitable to be used as a part of an embedded system. By using a small test database we have reached a success rate of $98.7 \%$.

Key words: image analysis, feature vectors, discrete wavelet transform
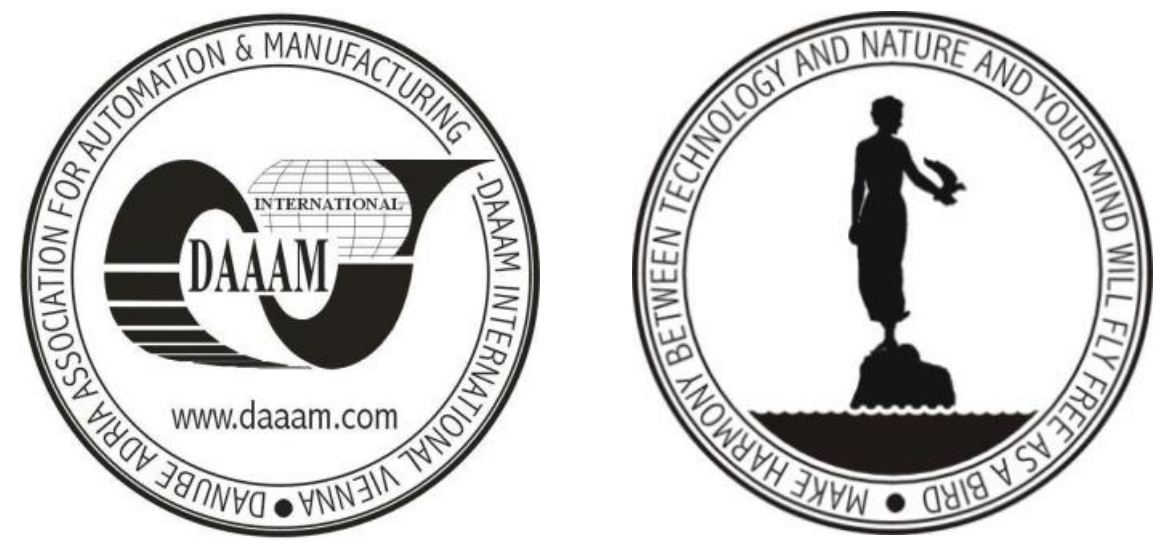

Authors' data: Dr. Sc. Rakun, J[urij]; Dipl.-Ing. Berk, P[eter]; Univ. Prof. Stajnko, $\mathrm{D}$ [enis]; M. Sc. Ocepek, M[arko]; Univ. Prof. Lakota, M[iran], Faculty of Agriculture and Life Scineces, University of Maribor, Pivola 10, 2311 Hoce, Slovenia, jurij.rakun@uni-mb.si, peter.berk@uni-mb.si, denis.stajnko@uni-mb.si, marko.ocepek@uni-mb.si,miran.lakota@uni-mb.si

This Publication has to be referred as: Rakun, J[urij]; Berk; P[eter]; Stajnko, D[enis]; Ocepek, M[arko] \& Lakota, M[iran] (2012), Digital Image Processing Approach to Fingerprint Authentication, Chapter 43 in DAAAM International Scientific Book 2012, pp. 517-526, B. Katalinic (Ed.), Published by DAAAM International, ISBN 978-3-901509-86-5, ISSN 1726-9687, Vienna, Austria DOI: $10.2507 /$ daaam.scibook.2012.43 
Rakun, J.; Berk, P.; Stajnko, D.; Ocepek, M. \& Lakota, M.: Digital Image Processi...

\section{Introduction}

In every day life we are often faced with a problem of how to protect crucial systems from unauthorized access. Usually only a small group of individuals has access to systems such as servers, bank machines, security systems and others. The usual approach is to provide each individual with a magnetic ID card, key or digital certificate. However the problem with this kind of authentication is that it can be copied or counterfeit, so its present does not guarantee that the user has the right to access the system. A good authentication method is therefor a method that uses keys that are hard or better yet impossible to copy, keys that cannot be transferred to other users and keys that can be used in any given time.

Instead of using a separate authentication objects, it is user-friendlier to analyse the characteristics of each user - its biometric properties. They mirror physiological characteristics, so they are unique to each individual and provide an ideal way of authentication. They cannot be forgotten, in most cases cannot be copied and do not change significantly with time. Such biometric markers can be established by analysing the speech, handwriting, iris patterns, distribution of blood vessels, DNK and fingerprints.

Each fingerprint can be represented as a quasi-periodic distribution of ridges and valleys. They are unique to each individual and develop in the uterus while the individual is still an embryo and do not change during its life. The fingerprints differ even between family members and even semi-identical twins. This is the reason the fingerprints offer a great, simple and non-invasive way of identifying an individual.

Different techniques to authenticate or analyse a fingerprint have been described by authors (Tyco et al., 2001), (Lee et al.,2001), (Bovik, 2005), (Espinosa et al., 2011) and (Simon-Zorita et al., 2001). They can be classified in on of two groups; the ones that use distinct points and the ones that relay on the whole fingerprint.

Each fingerprint is made up of patterns that are formed by ridges and valleys. They divide, merge and end in points called minutiae as described by (Espinosa et al., 2011) (Simon-Zorita et al., 2001), where each fingerprint has on average around 30 and 40. Based on the distribution of minutiae a unique identificator can be calculated. The comparison between these indetificators is fast but the procedure demands an extensive pre-processing of fingerprint images as shown by (Tyco et al., 2001) and (Bovik, 2005). Fig. 1 depicts three different fingerprint patterns.

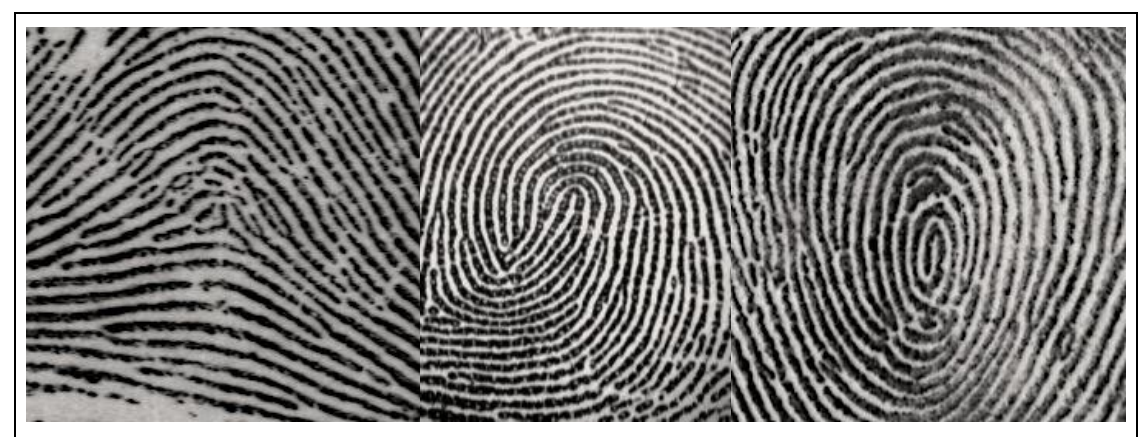

Fig. 1. Three different characteristic patterns (among others) that can be found in fingerprints (left: arch, middle: loop, second left: whorl) 
The second approach uses whole fingerprint images, as described by (Tyco et al., 2001) and (Bovik, 2005) and it is more suitable for images with lower degree of quality. The analysis based on the whole image is also more robust as it is less error prone when the fingerprints are moved or rotated in compared to the original. This can be solved by a pre-processing registration or correlation methods for the first group, but requires more system resources.

In this work we will show an algorithm we develop that can be classified partly in both groups. It uses the whole images, but it determines its own reference point that serves as a point of origin for the area of interest. As well, our goal was to develop an algorithm that would be fast enough to work on a system with limited resources, such as an embedded system.

\section{The algorithm}

Instead of using the whole fingerprint images, the algorithm constructs a unique feature vector for each of the fingerprints that are used to classify a new fingerprint to a set of familiar fingerprints. It works by applying the following steps: gradient calculation, minutiae detection, reference point calculation, area of interest detection, feature extraction based on discrete wavelet transform and finally feature comparison based on mid-squared error (MSE).

The fingerprint authentication algorithm starts by first calculating the gradients in $x$ and $y$ direction, based on which a gradient map (Gonzales et al., 2008) is calculated as depicted by the right side of Fig. 2 .

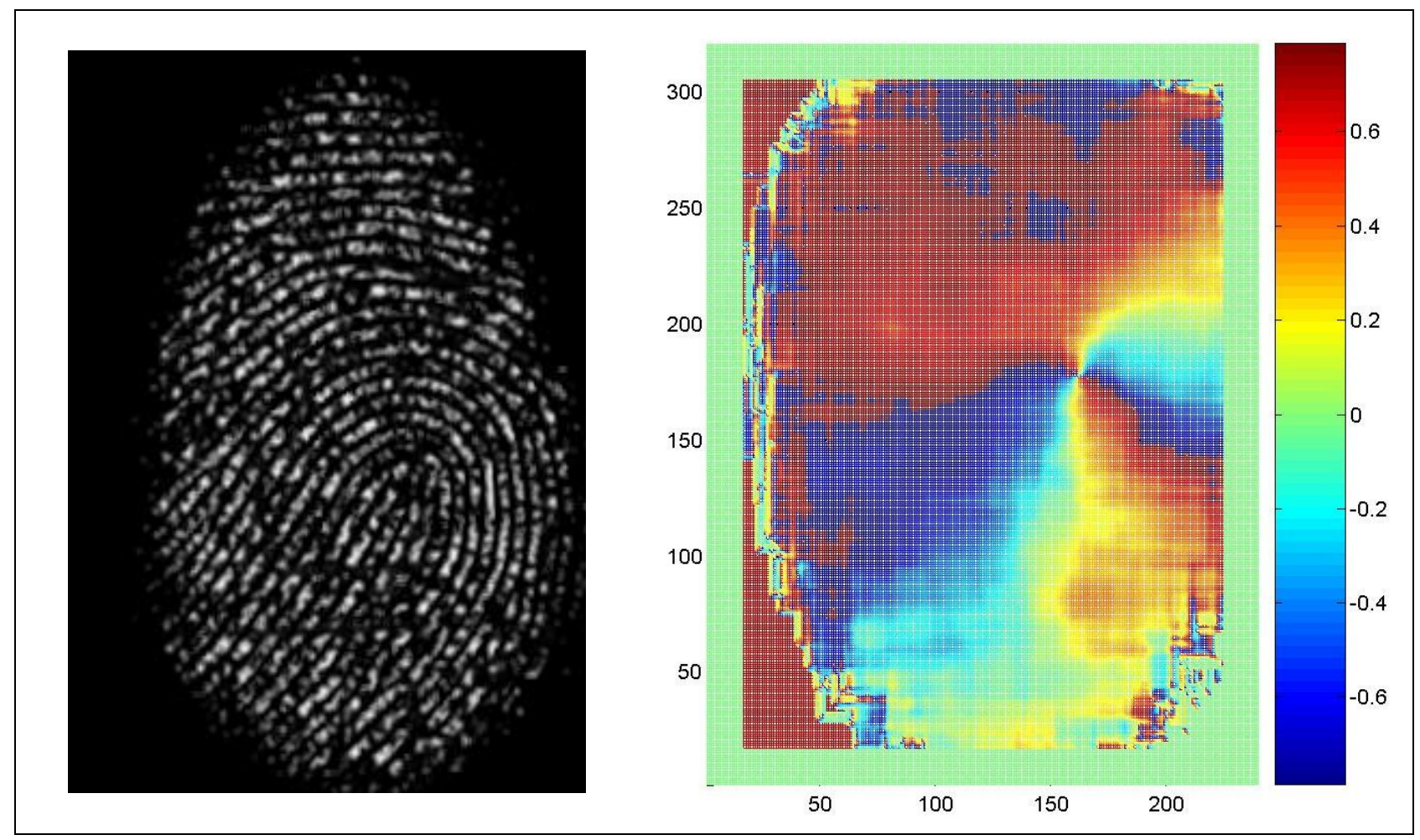

Fig. 2. An example of the input image with size of $240 \times 320$ pixels (left) and its gradient map (right) 
Rakun, J.; Berk, P.; Stajnko, D.; Ocepek, M. \& Lakota, M.: Digital Image Processi...

The left side image of Fig. 2 depicts an example fingerprint with one distinct minutia that shows up as a distinct extreme point on the right gradient map. The extreme point can be detected by looking for the whole interval of values from extreme positive to negative values in a limited area. Of course this can also reveal some false positive distinct extremes on the fingerprint borders that can affect the results and must be avoided.

The algorithm continuous with a binarisation step (Gonzales et al., 2008) revealing areas that are close to 0 . For our experiments we have set a fixed threshold interval between -0.2 and 0.2 that produces regions with value 1 and other, values higher or lover the interval, with value 0. For our example fingerprint from Fig. 2 this produces a result depicted by Fig. 3 (left). The result can be further cleaned up by using an erosion operator (Gonzales et al., 2008), which removes small irrelevant border regions. Then the image was segmented in to regions as depicted by the right image of Fig. 3. We can observe that the minutia is located just between the two detected regions. It can be detected, this time with no other doubtful areas, by calculating the shortest distance between all edge pixels of both regions. The distance map for is defined as a 2D matrix, that links together all edge pixels, represented by subsequent numbers. Edge pixels from the first region are grouped on the $X$-axis, while from the second on the $Y$-axis. It is depicted on Fig. 4.

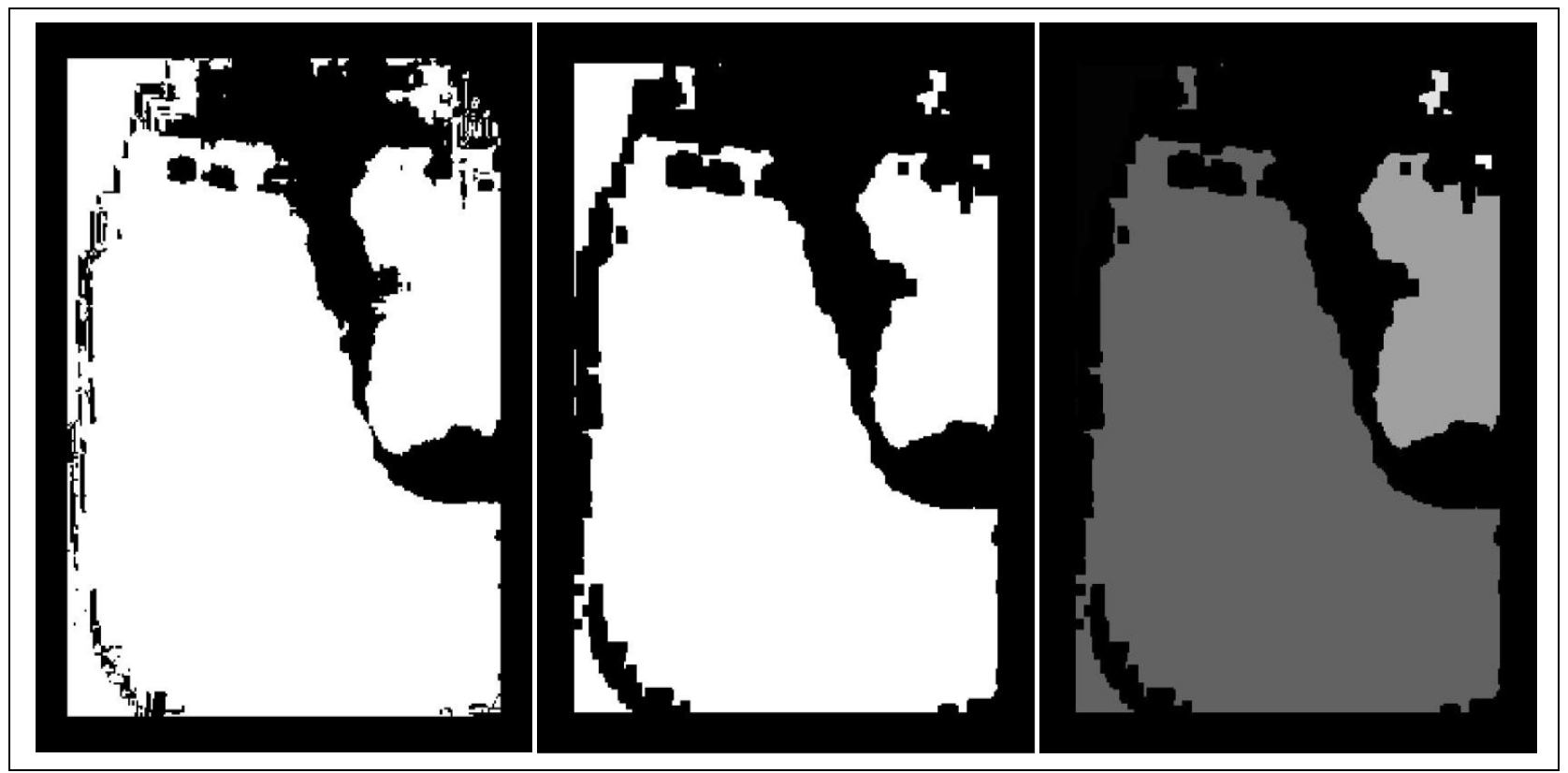

Fig. 3. Binarised image (left), its eroded (middle) and segmented version (right) 


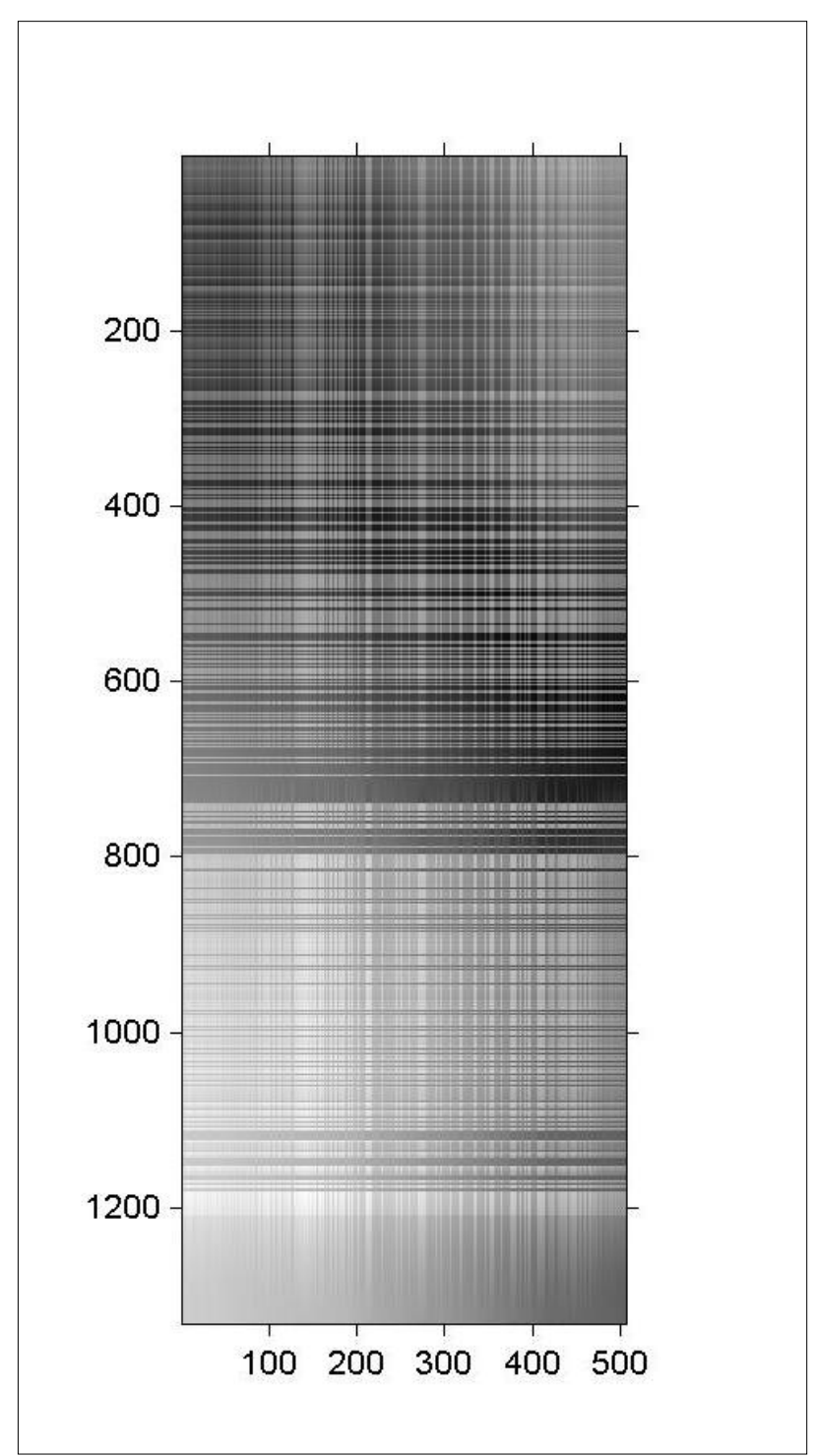

Fig. 4. Distance map for the two regions from Fig. 3

The minutia is detected with the help of distance map from Fig. 4. Its shows up as a global minimal value and its corresponding coordinates linking the two border pixels, one from one and the second from the other region. The location of the minutia is used as a reference point in the following stage of the algorithm.

As explained in the beginning, fingerprints differ one from another based on the pattern of arches, loops and whorls, as well as by the number of minutiae. In the first example we had one minutia, while in the examples on Fig. 6 fingerprints with more than one minutia are depicted. For these examples we proceed in the same manner as in the first example, but we construct multiple distance maps. The locations of the minutiae are then used to calculate their centre of gravity, which serves as a reference point for further analysis. 

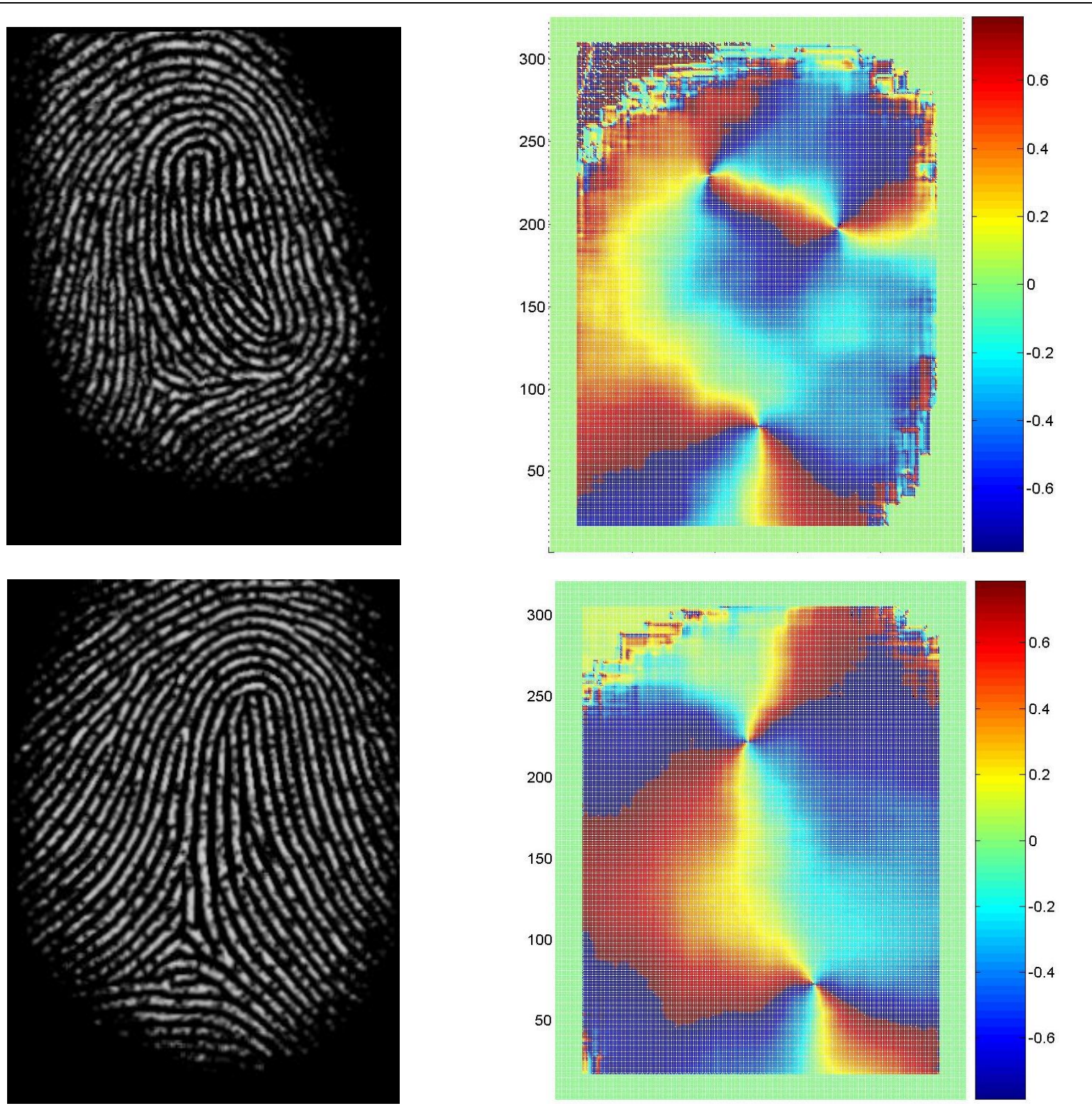

Fig. 5. Two different examples of fingerprints presented with their distance maps

Next, region of interest is selected in each direction around the reference point; left, right, up and down. The region size is adjusted to both compared fingerprint images and is extended to each direction for as long the energy is uniform. Once energy abruptions are detected in one of the images, the expansion of the region stops. The region of interest produces two windows around the reference point into both images, where both windows have the same size.

To extract the features from the fingerprints we have used a discrete wavelet transformation described by (Tyco et al., 2001) and (Gonzales et al., 2008). With its help an input image $I$ can be represented as a set of $3 J+1$ smaller images in the following manner:

$$
\left[i,\left\{p_{j}{ }^{l}, p_{j}^{2}, p_{j}^{3}\right\}\right],
$$

where image $i$ is an approximation to the input image with twice the lower resolution. The $p_{j}^{k}$ depicts details along the $X$-axis (for $\mathrm{k}=1$ ), $Y$-axis (for $\mathrm{k}=2$ ) and both together 
(for $\mathrm{k}=3$ ). In general the amplitude of the wavelet transform is increasing with each level of the transform. But based on (Tyco et al., 2001) we know this is not true for periodic images such as the one depicting fingerprint patterns. In case of fingerprints the energy changes with each level and can be lower or higher, independent to the previous level. This offers unique pattern based on which a fingerprint can be identified. We do not focus on the energy alone, but rather its standard deviation $\sigma_{j}^{k}$ that can be written with the octave $j$ in the form of three features as:

$$
\left[\sigma_{j}{ }^{1}, \sigma_{j}^{2}, \sigma_{j}^{3},\right]
$$

For the feature vector we have selected 4 octaves that produced a feature vector with 12 members per level. Fig. 6 depicts an example for the same fingerprint capture at different situations, along with its feature vectors. Further, Fig. 7 depicts two different fingerprints along with their future vectors so a visual comparison can be made on how the feature vectors differ from one to another.

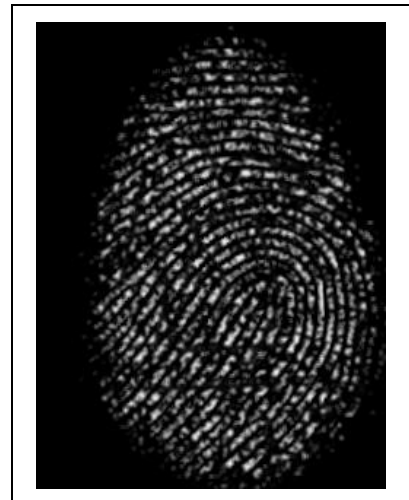

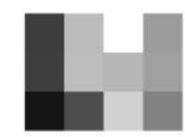

Feature vector for the left example.

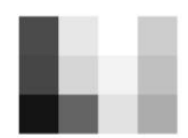

Feature vector for the right example.

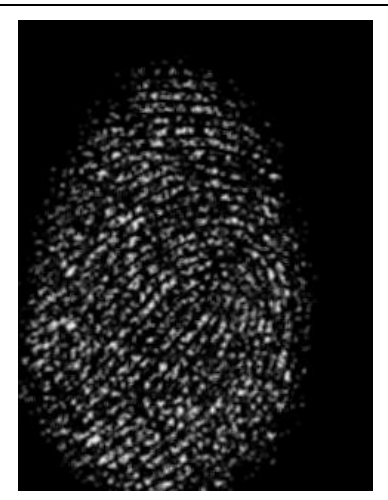

Fig. 6: Comparison of the same fingerprint captured at different situations, depicted along with their feature vectors.

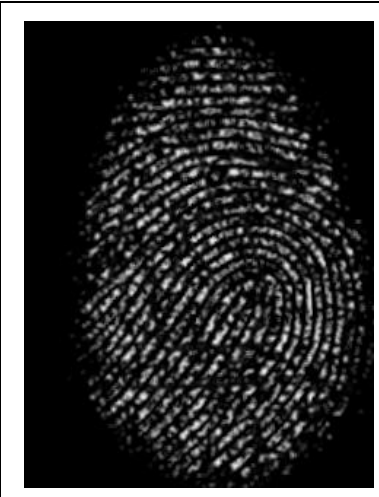

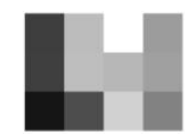

Feature vector for the left example.

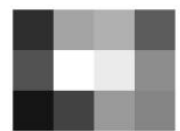

Feature vector for the right example.

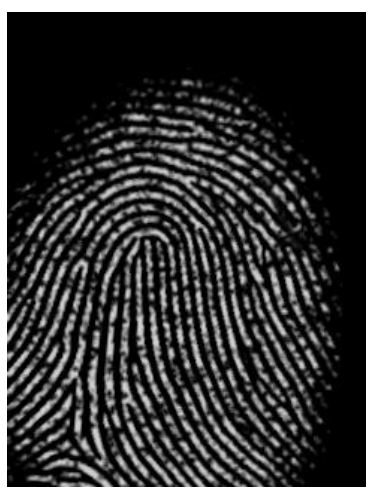

Fig. 7. Comparison of two different fingerprints, depicted along with their feature vectors

The final step of the algorithm is to compare feature vectors based on the midsquared-error (MSE). The pairs with the lowest MSE are the most similar and if the MSE is low enough it describes the same fingerprint. If the new fingerprint is close 
Rakun, J.; Berk, P.; Stajnko, D.; Ocepek, M. \& Lakota, M.: Digital Image Processi...

enough with one of the fingerprints in the database the algorithm authenticates the user.

\section{Results}

The results were measured using a small database of fingerprints provided by the Biometric System Lab. It included six different fingerprints, captured at 5 different situations. The images of the fingerprint had a resolution of $240 \times 320$ pixels. The fingerprint samples were presented as $x \_y$, where $y$ designated the number of the fingerprint and $x$ its iteration. For each of the fingerprints we extracted the feature vector and compared it to the feature vectors of all other fingerprints and their iterations. Comparison based on MSE for the first example is summarized in Tab. 1:

\begin{tabular}{|c|c|c|c|c|c|c|}
\hline $\begin{array}{c}\text { Comparison } \\
\text { 1_1 with: }\end{array}$ & 1_x & 2_x & 3_x & 4_x & 5_x & 6_x \\
\hline $\mathrm{x}=1$ & 0 & 0.0480 & 0.0473 & 0.0412 & 0.0441 & 0.0375 \\
\hline $\mathrm{x}=2$ & 0.0382 & 0.0470 & 0.0403 & 0.0437 & 0.0485 & 0.0436 \\
\hline $\mathrm{x}=3$ & 0.0304 & 0.0448 & 0.0532 & 0.0445 & 0.0473 & 0.0418 \\
\hline $\mathrm{x}=4$ & 0.0378 & 0.0456 & 0.0610 & 0.0430 & 0.0428 & 0.0461 \\
\hline $\mathrm{x}=5$ & 0.0311 & 0.0446 & 0.0587 & 0.0471 & 0.0434 & 0.0408 \\
\hline
\end{tabular}

Tab. 1. MSE based comparison of fingerprint 1_1 with all other fingerprints

By comparing a feature vector of fingerprint 1_1 with all others from the database we can conclude it reached the minimal MSE in the first set (with itself) as it to be expected. In case we would desire and set an empirical threshold value to 0.0382 and pronounce ones with lower or equal to that value to authenticated and those with higher to not authenticated we would get only one false positive or $96.7 \%$ success rate.

\begin{tabular}{|c|c|c|c|c|c|c|}
\hline $\begin{array}{c}\text { Comparison } \\
\text { 2_1 with: }\end{array}$ & 1_x & 2_x & 3_x & 4_x & 5_x & 6_x \\
\hline $\mathrm{x}=1$ & 0.0106 & 0 & 0.0092 & 0.0097 & 0.0114 & 0.0109 \\
\hline $\mathrm{x}=2$ & 0.0110 & 0.0068 & 0.0072 & 0.0105 & 0.0117 & 0.0120 \\
\hline $\mathrm{x}=3$ & 0.0129 & 0.0028 & 0.0142 & 0.0144 & 0.0136 & 0.0116 \\
\hline $\mathrm{x}=4$ & 0.0123 & 0.0030 & 0.0111 & 0.0128 & 0.0126 & 0.0093 \\
\hline $\mathrm{x}=5$ & 0.0095 & 0.0036 & 0.0147 & 0.0102 & 0.0130 & 0.0121 \\
\hline
\end{tabular}

Tab. 2. MSE based comparison of fingerprint 2_1 with all other fingerprints

Next, we compared fingerprint 2_1 with all other, as shown in Table 2. If we would decide and set a threshold of 0.0036 all test examples would be classified as true positives and we would reach a success ret of $100 \%$.

\begin{tabular}{|c|c|c|c|c|c|c|}
\hline $\begin{array}{c}\text { Comparison } \\
\text { 3_1 with: }\end{array}$ & 1_x & 2_x & 3_x & 4_x & 5_x & 6_x \\
\hline $\mathrm{x}=1$ & 0.0121 & 0.0092 & 0 & 0.0100 & 0.0118 & 0.0103 \\
\hline
\end{tabular}




\begin{tabular}{|c|c|c|c|c|c|c|}
\hline $\mathrm{x}=2$ & 0.0105 & 0.0095 & 0.0069 & 0.0090 & 0.0141 & 0.0100 \\
\hline $\mathrm{x}=3$ & 0.0153 & 0.0089 & 0.0088 & 0.0108 & 0.0118 & 0.0124 \\
\hline $\mathrm{x}=4$ & 0.0133 & 0.0086 & 0.0059 & 0.0134 & 0.0114 & 0.0107 \\
\hline $\mathrm{x}=5$ & 0.0106 & 0.0087 & 0.0122 & 0.0095 & 0.0115 & 0.0104 \\
\hline
\end{tabular}

Tab. 3. MSE based comparison of fingerprint 3_1 with all other fingerprints

The finger print 3_1 was compared in Table 3. Once again the majority of MSE based comparisons were successful. The only test case that proved to be more difficult is marked with 3_5, whit reaching its MSE up to 0.0122. A detailed analysis showed that example 3_5 had lower image quality, which affected the procedure of detecting reference points.

Next we tested examples $4 \_1,5 \_1$ and finally $6 \_1$ with all others and gathered the results in Table 4, Table 5 and Table 6 . In all test cases we managed to achieve $100 \%$ rate, by setting for the threshold the highest MSE value when comparing each fingerprint to the original group.

\begin{tabular}{|c|c|c|c|c|c|c|}
\hline $\begin{array}{c}\text { Comparison } \\
\text { 4_1 with: }\end{array}$ & 1_x & 2_x & 3_x & 4_x & 5_x & 6_x \\
\hline $\mathrm{x}=1$ & 0.0089 & 0.0097 & 0.0100 & 0 & 0.0102 & 0.0094 \\
\hline $\mathrm{x}=2$ & 0.0084 & 0.0090 & 0.0089 & 0.0062 & 0.0093 & 0.0112 \\
\hline $\mathrm{x}=3$ & 0.0121 & 0.0101 & 0.0115 & 0.0078 & 0.0131 & 0.0101 \\
\hline $\mathrm{x}=4$ & 0.0085 & 0.0092 & 0.0117 & 0.0081 & 0.0088 & 0.0097 \\
\hline $\mathrm{x}=5$ & 0.0102 & 0.0095 & 0.0133 & 0.0057 & 0.0098 & 0.0105 \\
\hline
\end{tabular}

Tab. 4. MSE based comparison of fingerprint 4_1 with all other fingerprints.

\begin{tabular}{|c|c|c|c|c|c|c|}
\hline $\begin{array}{c}\text { Comparison } \\
\text { 5_1 with: }\end{array}$ & 1_x & 2_x & 3_x & 4_x & 5_x & 6_x \\
\hline $\mathrm{x}=1$ & 0.0098 & 0.0114 & 0.0188 & 0.0102 & 0 & 0.0087 \\
\hline $\mathrm{x}=2$ & 0.0116 & 0.0103 & 0.0097 & 0.0089 & 0.0086 & 0.0097 \\
\hline $\mathrm{x}=3$ & 0.0123 & 0.0127 & 0.0109 & 0.0128 & 0.0069 & 0.0088 \\
\hline $\mathrm{x}=4$ & 0.0089 & 0.0131 & 0.0174 & 0.0120 & 0.0067 & 0.0112 \\
\hline $\mathrm{x}=5$ & 0.0098 & 0.0133 & 0.0111 & 0.0082 & 0.0074 & 0.0097 \\
\hline
\end{tabular}

Tab. 5. MSE based comparison of fingerprint 5_1 with all other fingerprints

\begin{tabular}{|c|c|c|c|c|c|c|}
\hline $\begin{array}{c}\text { Comparison } \\
\text { 6_1 with: }\end{array}$ & 1_x & 2_x & 3_x & 4_x & 5_x & 6_x \\
\hline $\mathrm{x}=1$ & 0.0079 & 0.0109 & 0.0103 & 0.0094 & 0.0087 & 0 \\
\hline $\mathrm{x}=2$ & 0.0057 & 0.0091 & 0.0105 & 0.0084 & 0.0111 & 0.0050 \\
\hline $\mathrm{x}=3$ & 0.0100 & 0.0100 & 0.0132 & 0.0110 & 0.0112 & 0.0054 \\
\hline $\mathrm{x}=4$ & 0.0099 & 0.0099 & 0.0114 & 0.0121 & 0.0093 & 0.0067 \\
\hline $\mathrm{x}=5$ & 0.0071 & 0.0098 & 0.0117 & 0.0076 & 0.0083 & 0.0059 \\
\hline
\end{tabular}

Tab. 6. MSE based comparison of fingerprint 6_1 with all other fingerprints 
Rakun, J.; Berk, P.; Stajnko, D.; Ocepek, M. \& Lakota, M.: Digital Image Processi...

By analysing the results of all test runs, we can conclude that the presented algorithm produced two false positives out of 150 test runs, giving an overall success rate of $98.7 \%$.

\section{Conclusion}

In the previous sections we have presented an algorithm for fingerprint verification that helps to identify an individual and works with the help of multiple digital image processing techniques. The procedure is simple enough, consisting of 7 straightforward image processing techniques, and is therefor suitable to be used on an embedded device. By comparing a representative of each fingerprint to all other fingerprints in our database we have reached an overall success rate of $98.7 \%$.

Multiple steps could be tested to further improve the algorithm. As suggested by the discussion in section four, one crucial improvement would be to change the procedure of detecting the reference point, which is not perfect. This could be done with the help of template matching of minutia using phase correlation approach, which is unaffected by the translations and rotations and will be tested and discussed in our future publications.

\section{References}

Biometric System Lab.: http://biolab.csr.unibo.it/databasesoftware.asp Accessed: 23.4.2012

Bovik, A. (2005), The Handbook of image and video processing, 2 ed., pp. 12191233, Elsevier, New York

Espinosa, V. (2001), Minutiae detection algorithm for fingerprint recognition, IEEE 35th International Carnahan Conference on Security Technology, pp. 264 266

Gonzales, R. C. \& Woods, R. E. (2008), Digital Image Processing, $3^{\text {rd }}$ edition, Prentice Hall, Upper Saddle River, NJ, USA

Jiang, X. M.; Liu, A. \& Kot, C. (2004), Reference point detection for fingerprint recognition, ICPR 2004 - 17th International Conference on Pattern Recognition, vol. 1, pp. $540-543$

Lee, C.-J.; Wang, S.-D. \& Wu, K.-P. (2001), Fingerprint recognition using principal Bagor basis function, Procedings of 2001 International Symposium on Inteligent Multimedia, Video and Speech Processing, pp. 393 - 396, Hong Kong

Simon-Zorita, D.; Ortega-Llanas, J. \& Gonzales-Rodriguez, J. (2001), Minutiae extraction scheme for fingerprint recognition systems, International Conference on Image Processing, vol. 3., pp. $254-257$

Tico, M.; Immonen, E.; Rämö, P.; Kuosmanen, P. \& Saarinen, J. (2001), Fingerprint recognition using Wavelet features, ISCAS 2001 - The 2001 IEEE International Symposium on Circuits and Systems, vol. 2, pp. $21-24$ 